\title{
Efficient CFIE-MOM Analysis of 3-D PEC Scatterers in Layered Media
}

\author{
Kim, Oleksiy S.; Jørgensen, E.; Meincke, Peter; Breinbjerg, Olav
}

Published in:

Proceedings of International Conference on Mathematical Methods in Electromagnetic Theory, Kiev, Ukraine

Publication date:

2002

Document Version

Publisher's PDF, also known as Version of record

Link back to DTU Orbit

Citation (APA):

Kim, O. S., Jørgensen, E., Meincke, P., \& Breinbjerg, O. (2002). Efficient CFIE-MOM Analysis of 3-D PEC

Scatterers in Layered Media. In Proceedings of International Conference on Mathematical Methods in Electromagnetic Theory, Kiev, Ukraine (pp. 476-478). IEEE.

\section{General rights}

Copyright and moral rights for the publications made accessible in the public portal are retained by the authors and/or other copyright owners and it is a condition of accessing publications that users recognise and abide by the legal requirements associated with these rights.

- Users may download and print one copy of any publication from the public portal for the purpose of private study or research.

- You may not further distribute the material or use it for any profit-making activity or commercial gain

- You may freely distribute the URL identifying the publication in the public portal 


\title{
EFFICIENT CFIE-MOM ANALYSIS OF 3-D PEC SCATTERERS IN LAYERED MEDIA
}

\author{
Oleksiy S. Kim, Erik Jørgensen, Peter Meincke, and Olav Breinbjerg \\ Ørsted•DTU, Electromagnetic Systems, Technical University of Denmark, \\ Building 348, Ørsteds Plads, DK-2800 Kgs. Lyngby, Denmark \\ Tel: +45 4525 3800, Fax: +45 4593 1634, E-mail: osk@oersted.dtu.dk
}

\begin{abstract}
This paper presents an efficient technique for analysis of arbitrary closed perfectly conducting (PEC) scatterers in layered media. The technique is based on a method of moments (MoM) solution of the combined field integral equation (CFIE). The high efficiency is obtained by employing an accurate expansion of the multilayer dyadic Green's function along with a higher order hierarchical discretization scheme and a rapidly converging iterative solver with preconditioner.
\end{abstract}

\section{INTRODUCTION}

Numerical analysis of electromagnetic scattering by PEC objects has attracted great attention for many years. Naturally, available computational resources limit the size of objects that can be analysed by numerical techniques. The situation becomes even worse when layered medium is considered. Furthermore, for objects penetrating the interface (interfaces) between the layers, conventional methods can not efficiently handle the different wavelength in different media.

In this paper, a new efficient MoM scheme, combining fast calculation of dyadic Green's functions for multilayered media with higher order hierarchical basis functions, is presented. This technique allows the number of unknowns to be decreased in comparison with conventional techniques employing low-order discretization schemes. The relaxation of memory requirements is especially pronounced when the scatterer is located in two or more layers. Further improvement in computational efficiency is achieved by employing a rapidly converging iterative solver with preconditioner. The technique is validated with examples available in the literature.

\section{INTEGRAL EQUATION TO BE SOLVED}

The electric field integral equation (EFIE) and the magnetic field integral equation (MFIE) each fail at a set of discrete frequencies for closed PEC objects. Being a linear combination of EFIE and MFIE, CFIE provides stable and reliable solutions at all frequencies. EFIE is a well-known and quite elaborated technique for analysis of scattering by PEC objects both in free space and in layered media. At the same time, MFIE is usually applied for scatterers in homogeneous media. There are very few papers devoted to MFIE in layered media and only conducting bodies of revolution have been treated thoroughly by MFIE [1] (the MFIE-BOR method). In this paper, we apply the MFIE (as an essential part of CFIE) to arbitrary closed PEC objects in layered media. 


\section{DYADIC GREEN'S FUNCTIONS FOR MULTILAYERED MEDIA}

The dyadic Green's functions for multilayered media are implemented using the Formulation $\mathrm{C}$ for the mixed potential integral equation [2]. Under this formulation, only three different Sommerfeld integrals arise in the solution. They are computed in a very efficient way using the discrete complex image method (DCIM) [3] which allows spatial Green's functions to be represented in closed form in terms of complex images. If the scatterer is confined to one layer, the coefficients of the complex image expansion are invariant with respect to the coordinates of the source and observation points. Otherwise, the coefficients depend only on the z-coordinate of the source point and the layer the observation point belongs to. It is worth noting that the coefficients of the complex image expansion are reused in both EFIE and MFIE.

\section{DISCRETIZATION TECHNIQUES}

Higher order hierarchical basis functions [4] based on orthogonal Legendre polynomials are employed for discretizing the CFIE. The hierarchical property of the functions provides a very efficient discretization scheme especially when the scatterer is not confined to one layer. The order of the polynomial expansion can be selected separately on each patch depending on the wavelength of the layer in which the patch is located. This allows a very low number of unknowns and a uniform mesh despite the different wavelengths. In contrast to this, conventional low-order methods are forced to use a patch size based on the shortest wavelength, which introduces unnecessary unknowns and leads to a poor condition number of the resulting MoM matrix. Alternatively, conventional methods may employ a highly non-uniform mesh that is difficult to construct and also leads to a poor condition number. The higher order hierarchical basis functions are here employed on second-order curved patches that provide accurate geometrical modeling of smooth surfaces.

\section{IMPLEMENTATION AND NUMERICAL RESULTS}

The described technique has been implemented in an efficient computer code that is well parallelized on both shared and distributed memory multiprocessor systems. The most time consuming part of the analysis is the calculation of the dyadic Green's functions for multilayered media. On a multiprocessor workstation, it can be done in parallel for different pairs of source and observation points. Furthermore, due to reusing of the Green's function expansion in both EFIE and MFIE, the CFIE matrix filling time increases only by $10 \%$ with respect to MFIE alone.

The presented technique was validated by comparison with the results obtained by the MFIE-BOR method [1] for scattering by a PEC sphere in the presence of the interface between two contiguous half-spaces. For instance, Fig. 1 shows the currents on a halfburied metallic sphere illuminated by a plane wave. It is observed that the results of the new technique are in excellent agreement with those of [1]. Fig. 2 presents a new result for a geometry that can not be treated by the MFIE-BOR method [1]. Both a first-order discretization scheme (rooftops on a uniform mesh) and a hierarchical discretization scheme are used. In the first case, significant overdiscretization in the free space is observed (709 unknowns per square wavelength, see Table 1). In the second case, the

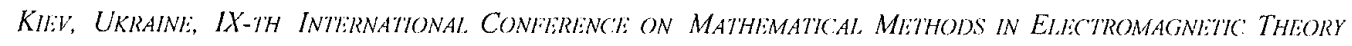


hierarchical discretization allows the total number of unknowns to be reduced by more than a factor of three while the accuracy is maintained (Fig. 2).

\section{REFERENCES}

[1] A.A.K. Mohsen et al., Progress In Electromag. Research, PIER 24, pp. 19-37, 1999.

[2] K.A. Michalski et al., IEEE Trans., vol. AP-38, pp. 335-344, 1990.

[3] Y.L. Chow et al., IEEE Trans., vol. MTT-39, pp. 588-592, 1991.

[4] E. Jørgensen et al., Proc. of IEEE AP-S, San Antonio, USA, 2002, pp. 618-621.
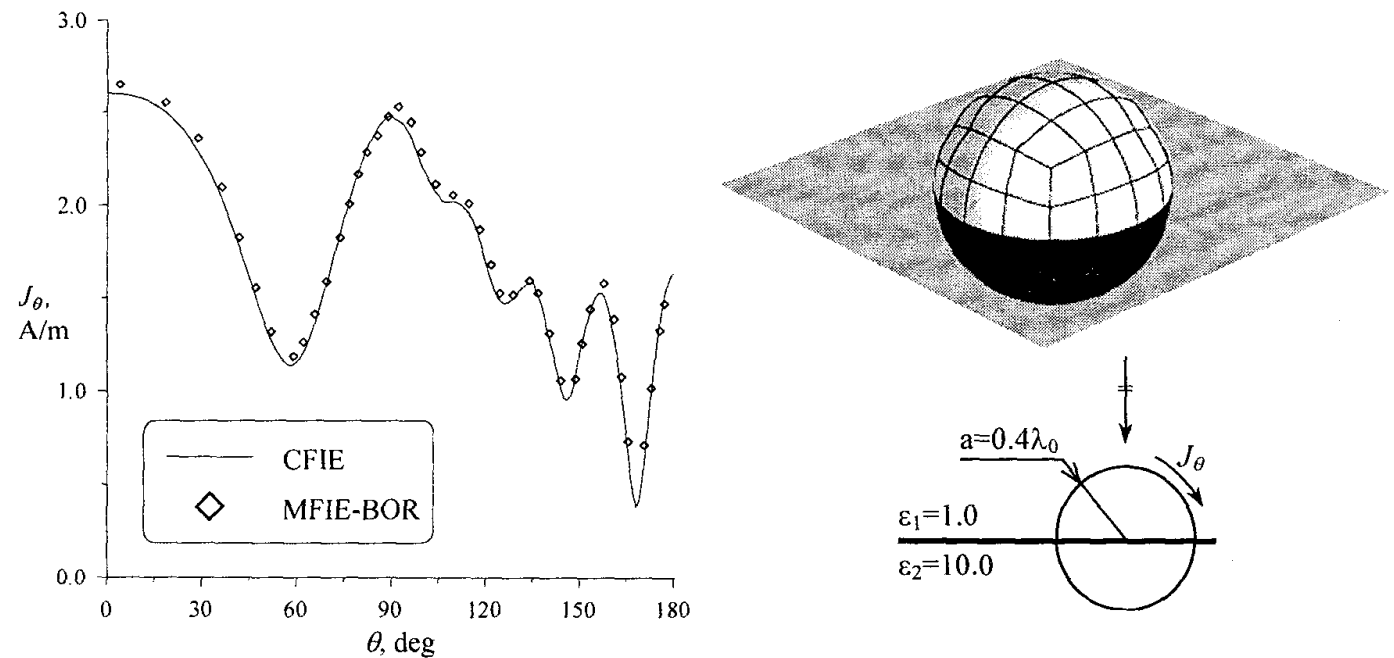

Fig. 1. Current on the half-buried PEC sphere illuminated by a plane wave.

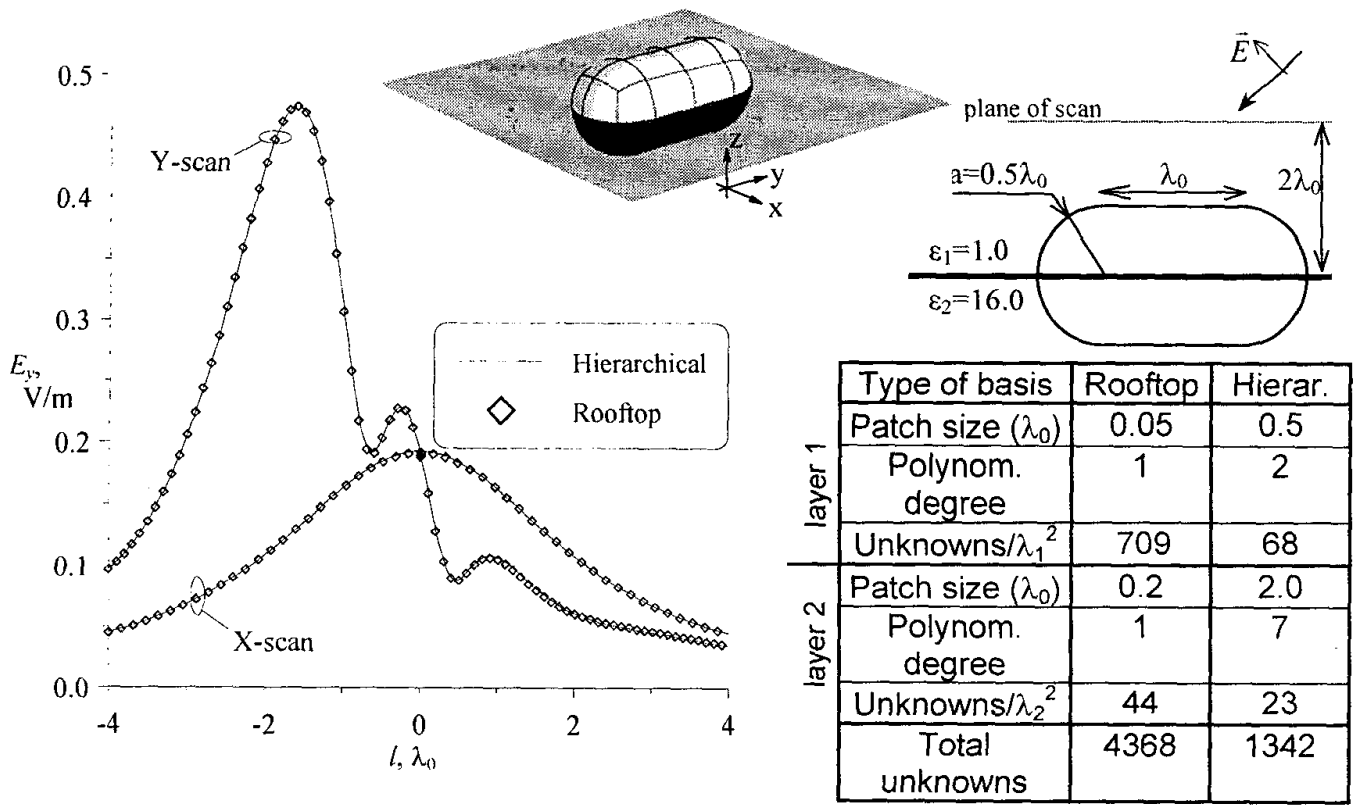

Fig. 2. Scattered near-field from the half-buried PEC tube illuminated by a plane wave. 